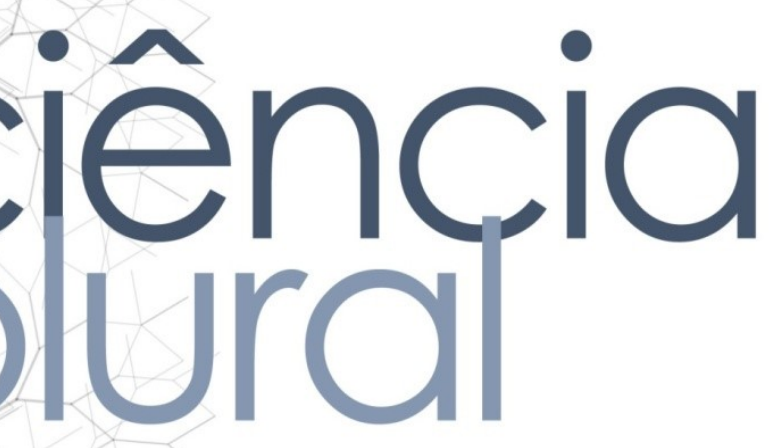

\title{
AUTOPERCEPÇÃO SOBRE SAÚDE BUCAL E SUA RELAÇÃO COM UTILIZAÇÃO DE SERVIÇOS E PREVALÊNCIA DE DOR DE DENTE
}

\section{Self-perception on oral health and its relationship with use of services and prevalence of tooth ache}

Letícia Mendes Santos • Graduada em Odontologia pela Universidade Federal do Rio Grande do Norte. E-mail: leticia_mendes93@hotmail.com

Luiz Roberto Augusto Noro • Doutor em Ciências da Saúde. Professor Adjunto IV do Departamento de Odontologia / Universidade Federal do Rio Grande do Norte. E-mail: noro@ufrnet.br

Angelo Giuseppe Roncalli - Doutor em Odontologia Preventiva e Social. Professor Associado III do Departamento de Odontologia / Universidade Federal do Rio Grande do Norte. E-mail: roncalli@terra.com.br

Ana Karine Macedo Teixeira • Doutora em Saúde Coletiva. Professora Adjunto I do Curso de Odontologia I Universidade Federal do Ceará. E-mail: anakarinemt@hotmail.com

Autora responsável pela correspondência:

Letícia Mendes Santos. E-mail: leticia_mendes93@hotmail.com 



\title{
RESUMO
}

Introdução: Autopercepção em saúde abrange a compreensão que o indivíduo possui sobre o seu próprio estado de saúde, baseando-se em experiências anteriores e no contexto social, cultural e histórico em que está inserido. Objetivo: Analisar a autopercepção em saúde bucal de adolescentes e adultos jovens com 17-21 anos no município de Sobral-CE, relacionando-a com a utilização de serviços odontológicos e prevalência de dor de dente. Métodos: Estudo transversal aninhado à terceira onda da coorte de saúde bucal, desenvolvida no ano de 2012, com 482 participantes, a partir de questionário validado pelo Projeto SB Brasil 2010. Resultados: Houve associação entre classificação da saúde bucal autorreferida com mastigação, aparência de dentes e gengiva e necessidade de tratamento. Além disso, autopercepção sobre a mastigação teve associação com dor de dente e a necessidade de tratamento autorreferida com a utilização de serviços e dor de dente. Conclusões: A percepção sobre saúde bucal de adolescentes e adultos jovens está relacionada com a estética, função mastigatória e a necessidade de tratamento. Esse quadro sinaliza a preferência da utilização dos serviços odontológicos em casos de dor em detrimento de procedimentos preventivos. Seria importante que o planejamento em saúde bucal fosse orientado não somente por dados normativos, mas que incluísse a percepção da população para que as ações propostas fossem mais coerentes e aceitáveis pelos usuários.

Palavras-chave: Saúde bucal; Autoimagem; Assistência Odontológica.

\begin{abstract}
Introduction: Self-perceived health is related to the health knowledge that individuals have about their own status based on both previous experiences and the social, cultural and historical context in which they are inserted. Objective: To analyze the self-perception of oral health of adolescents and young adults aged between 17-21 years in Sobral-CE and its relation to the use of dental services and prevalence of toothache. Methods: Cross-sectional study nested to the third wave of an oral health cohort, developed in 2012, with 482 participants aged between 17-21 years, with a questionnaire validated by a National Oral Health Survey in Brazil, the Project SB Brasil 2010. Results: There was association between self-reported rating of oral health with chewing, appearance of teeth and gums and treatment need. In addition, mastication perception was directly associated with toothache and self-reported need treatment was associated with the use of services and toothache. Conclusions: The perception about oral health in adolescents and young adults is related to aesthetics, masticatory function and treatment need. This situation indicates the preference by using dental services in cases of toothache instead of preventive procedures. It is important that oral health planning services should not only be guided by normative data, but includes the perception of the population so that the proposed actions would be more consistent and acceptable from patients.
\end{abstract}

Keywords: Oral health; Self Concept; Dental care. 


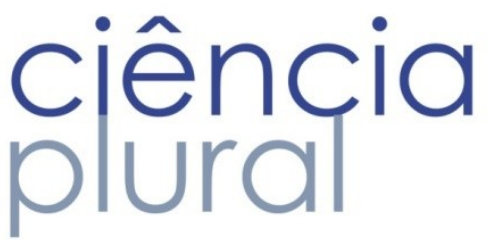

Introdução

A autopercepção em saúde abrange a compreensão que 0 indivíduo possui sobre 0 seu estado de saúde, englobando elementos subjetivos relacionados ao seu bem-estar ${ }^{1}$. Refere-se à maneira pela qual 0 sujeito compreende suas próprias atitudes e crenças com base em seu comportamento em determinadas situações, mediadas pelos diversos aspectos de sua saúde física, cognição e capacidade funcional2. Esse discernimento baseia-se no conhecimento dessa pessoa sobre o processo saúde-doença, que se encontra interligado às suas experiências anteriores e pelo contexto social, cultural e histórico em que está inserido 3 .

A concepção dos pacientes sobre esses componentes físicos e emocionais vão afetar diretamente seu comportamento em relação aos cuidados com a saúde. Estudos afirmam que a razão para muitos não procurarem os serviços odontológicos é a sua percepção de que não necessitam de tratamento 4 .

É importante, ainda, salientar a correlação entre a autopercepção e os fatores socioeconômicos, visto que estudos asseguram que a pior autoavaliação (pessoas que não conseguem identificar sua condição de saúde mais aproximada da real condição) associa-se diretamente com indicadores de iniquidades sociais. ${ }^{4}$

Sendo assim, é imprescindível analisar os elementos que levam a satisfação com a saúde bucal e não somente a condição clínica do paciente observada por levantamentos epidemiológicos, proporcionando uma maior compreensão de como se deve organizar um tratamento odontológico eficiente e satisfatório no ponto de vista do paciente. Além disto, verificar o acesso a serviços odontológicos ajuda a compreender um pouco mais a relevância da autopercepção em saúde bucal para a procura e utilização desses serviços.

Nesse sentido, o presente estudo objetiva analisar a autopercepção em saúde bucal de adolescentes e adultos jovens entre 17 e 21 anos no município de Sobral-CE, relacionando-a com a utilização de serviços odontológicos e dor de dente.

\section{Metodologia}

Estudo observacional com desenho seccional, aninhado a uma coorte que teve início no ano 2000, com ondas nos anos de 2006 e 2012. O local de estudo foi o município de Sobral-CE, que se situa no norte do estado do Ceará a $235 \mathrm{~km}$ da capital, Fortaleza. Segundo o IBGE 5 sua população estimada em 2015 é de 201.756 habitantes e a área de unidade territorial é de 2.122,897 km². O Índice de Desenvolvimento Humano Municipal-2010 (IDHM-2010) é de 0,714 e o produto interno bruto per capita a preços correntes em 2012 era de 


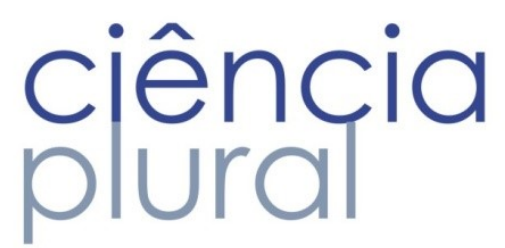

$R \$ 12.750,83$. Dos 50.445 domicílios particulares permanentes em 2010, apenas 9.079 possuíam automóveis para uso particular, 711 domicílios tinham como material de suas paredes externas apenas taipa e $1684 \mathrm{com}$ taipas revestidas. Além disso, Sobral conta com 69 estabelecimentos de saúde do Sistema Único de Saúde $(\mathrm{SUS})^{5}$.

Em 2012 foi realizada busca ativa da população examinada em 2006 (688 indivíduos), inicialmente junto as Unidades de Saúde da Família (USF), nas quais foram entregues listas dos jovens que residiam na área de abrangência de cada unidade. A identificação dos jovens que ainda ali residiam foi feita pelos Agentes Comunitários de Saúde (ACS) que os convidaram a comparecer em um dia agendado na unidade de saúde mais próxima para que fosse realizada a avaliação. A fim de maximizar a quantidade de pessoas abrangidas, para aqueles que não compareceram no dia e hora marcados no USF, a equipe de campo realizou até três visitas domiciliares em horário diurno.

E para os indivíduos que não residiam mais no mesmo endereço, com intuito de minimizar as perdas dos participantes, foram utilizadas informações sobre o novo endereço relatado por vizinhos, parentes e ACS. Realizaram-se também visitas em escolas públicas, visita a uma fábrica de calçados conhecida no município por empregar muitos jovens na faixa etária estudada, além de uma busca ativa nas redes sociais como Facebook.

Com essa metodologia de busca foi possivel obter uma taxa de resposta de $70 \%$ resultando em uma amostra de 482 jovens na faixa etária de 17 a 21 anos. A perda desses $30 \%$ ocorreu por mudança de endereço (24), recusa em participar (10), preso (8), morte (3) e morador de rua (1).

A metodologia aplicada para a coleta de dados clínicos foi baseada nos critérios da Organização Mundial de Saúde (OMS) para levantamentos epidemiológicos em saúde bucal6. Acrescido a isso, foi realizada uma entrevista com os participantes, com base em um questionário utilizado para a coleta de dados do Projeto SB Brasil 2010?.

A coleta dos dados foi realizada por sete equipes de estudantes do último ano do curso de Odontologia da Universidade Federal do Ceará - Campus Sobral devidamente capacitados, sob supervisão da pesquisadora principal do estudo. Foi realizada a calibração interexaminadores pela técnica de consenso, com o índice Kappa variando de 0,80 a 0,93 . Os exames ocorreram sob luz natural, com uso de espelho e sonda periodontal da OMS. ${ }^{8}$ 


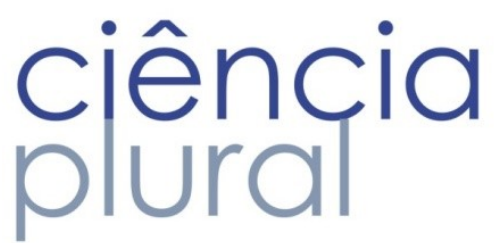

Esse questionário continha perguntas divididas nas seguintes categorias: condições sociodemográficas (sexo, raça, escolaridade, estudante, tipo de escola, moradia, aglomeração familiar, trabalho, tipo de trabalho, renda familiar, participação em grupo de adolescentes, abastecimento de água); hábitos e comportamentos de saúde bucal (escova dental, dentifrício, fio dental e frequência de escovação); autopercepção de saúde bucal (classificação da saúde bucal, aparência, mastigação e fala, e, se a saúde bucal afeta relacionamentos); ações de saúde bucal (utilização dos serviços odontológicos pelo menos uma vez na vida, tempo, local e motivo da última consulta odontológica, acesso negado, uso de aparelho ortodôntico, causa de traumatismo dentário) e morbidade autorreferida (dor de dente, necessidade de tratamento).

Para esse estudo, foram analisadas as variáveis referentes à autopercepção de saúde bucal, ações de saúde bucal e morbidade autorreferida. As variáveis relativas à autopercepção em saúde bucal foram dicotomizadas em situação adequada (boa ou ótima) e condição inadequada (ruim ou péssima). As ocorrências que foram classificadas como regular foram consideradas "missing".

Nas variáveis relativas às ações de saúde bucal, foi considerada adequada a utilização de serviços odontológicos ao menos uma vez na vida, assim como a última visita ao dentista ter sido realizada a menos de um ano.

A análise dos dados foi realizada por meio de digitação dupla, a fim de corrigir possíveis inconsistências. Para a construção do banco de dados utilizou-se o software de construção de planilhas eletrônicas Excel. A análise estatística, por sua vez, foi realizada com o uso do programa Stata 14. Para analisar a relação entre as variáveis dependentes e independentes foi realizado o teste de associação do Qui-quadrado.

Em relação aos aspectos éticos, a pesquisa foi submetida e aprovada pelo Comitê de Ética em Pesquisa da Universidade Estadual Vale do Acaraú (UVA) com protocolo de número 1019 - processo 430055, CAE: 0067.0.039.000-11, aprovado em 3 de fevereiro de 2012. Todos os participantes assinaram Termo de Consentimento Livre e Esclarecido (TCLE) e os menores de idade foram autorizados a participar da pesquisa pelos responsáveis.

\section{Resultados}

O presente estudo contou com um total de 482 participantes, sendo $51,9 \%$ do sexo feminino com faixa etária distribuída da seguinte forma: 116 possuíam 17 anos, 86 com 18 anos, 94 aos 19 anos, 90 com 20 anos e 96 com 21 anos. 


\section{ciência plural}

Pela análise das variáveis independentes foi possível constatar que $76,8 \%$ dessa população estudavam e/ou trabalhavam. Dos estudantes, $81,7 \%$ frequentavam a escola pública e dos que trabalhavam $58,3 \%$ possuíam vínculo trabalhista (carteira assinada ou estatutário). Quando considerada a escolaridade do chefe da família, apenas 10,7\% tinham ensino médio ou superior, 7,9\% possuíam ensino fundamental completo, $60,7 \%$ tinham ensino fundamental incompleto (até o $5^{\circ}$ ano) e 20,7\% não tiveram acesso a educação formal. A renda média familiar relatada foi de $\mathrm{R} \$ 1037,97$ e a maioria relatou residir em casa própria $(81,7 \%)$.

Em relação às variáveis relacionadas à autopercepção em saúde bucal obteve-se que $74,7 \%$ dos indivíduos classificaram sua saúde bucal como boa ou ótima (adequada), 90,8\% acreditam mastigar adequadamente, $75,1 \%$ avaliaram que a aparência dos seus dentes e gengiva também estava adequada, e apenas $0,9 \%$ consideraram que sua fala se encontrava prejudicada pela sua condição bucal. Havendo, assim, uma preponderância da autoavaliação positiva da condição da boca. Todavia, 48,5\% relataram que 0 seu relacionamento interpessoal era afetado pela sua saúde bucal e $66,7 \%$ afirmaram necessitar de tratamento odontológico.

Após análise univariada entre a classificação da saúde bucal autorreferida e as variáveis relativas à autopercepção em saúde bucal, foram observadas associações conforme apresentado na Tabela 1.

Tabela 1: Associação entre classificação da saúde bucal autorreferida e outras variáveis relativas à autopercepção em saúde bucal e necessidade de tratamento em adolescentes e adultos jovens, Sobral - CE, 2012.

\begin{tabular}{lcccccc}
\hline \multicolumn{1}{c}{$\begin{array}{c}\text { Classificação SB } \\
\text { autorreferida }\end{array}$} & \multicolumn{2}{c}{ Adequada } & \multicolumn{2}{c}{ Inadequada } & \\
Variáveis & $\mathbf{n}$ & $\%$ & $\mathbf{n}$ & $\%$ & $\boldsymbol{P}$ \\
\hline Mastigação & & & & & \\
& Adequada & 177 & 79,0 & 47 & 21,0 & $<0,001$ \\
Inadequada & 11 & 37,9 & 18 & 62,1 & \\
Aparência de dentes e gengiva & & & & & \\
Adequada & 164 & 90,6 & 17 & 9,4 & $<0,001$ \\
Inadequada & 18 & 32,1 & 38 & 67,9 & \\
Relacionamento Interpessoal & & & & & \\
Não afeta & 117 & 79,1 & 31 & 20,9 & 0,106 \\
Afeta & 80 & 69,6 & 35 & 30,4 & \\
Necessidade tratamento autorreferida & & & & & \\
Não & 117 & 94,4 & 7 & 5,6 & $<0,001$ \\
Sim & 86 & 58,1 & 62 & 41,4 & \\
\hline
\end{tabular}




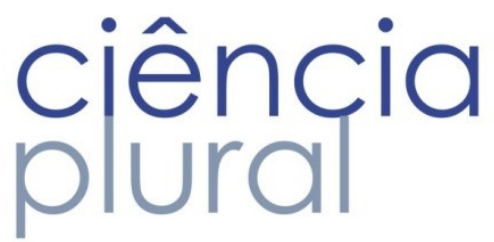

Foram observadas associações significativas da classificação da saúde bucal autorreferida com mastigação, aparência de dentes e gengiva e necessidade de tratamento. Apenas a variável correspondente à influência da saúde bucal no relacionamento interpessoal não se apresentou estatisticamente significativa.

Já em relação aos aspectos relativos ao serviço odontológico constatou-se que 7,5\% dos participantes da pesquisa não tinham ido ao dentista nenhuma vez na vida. Dos que utilizaram o serviço odontológico 90,3\% foi em serviços públicos e a avaliação do atendimento foi positiva para $95,5 \%$ desses indivíduos.

Entre os participantes que já tiveram acesso ao dentista, 49,8\% haviam feito essa visita há mais de um ano e 0 acesso negado por algum motivo foi relatado em $24,7 \%$ dos casos, sendo que $92,3 \%$ dessas situações foram em serviços públicos. Além disso, dentre os participantes do estudo $73 \%$ alegam já ter sentido dor de dente alguma vez na vida e dentre esses, apenas $27,4 \%$ afirmaram que o primeiro procedimento que realizaram foi procurar um dentista. Em 33\% dos participantes a intensidade da dor causada pelos dentes e gengivas nos últimos seis meses foi de média a muito alta. A associação entre aparência dos dentes e gengiva com utilização do serviço odontológico, tempo da última visita ao dentista e dor de dente é demonstrada na Tabela 2.

Tabela 2: Associação entre aparência de dentes e gengiva e utilização do serviço odontológico, tempo da última visita ao dentista e dor de dente em adolescentes e adultos jovens, Sobral - CE, 2012.

\begin{tabular}{|c|c|c|c|c|c|c|}
\hline \multirow[b]{2}{*}{ Variáveis } & \multirow{2}{*}{$\begin{array}{r}\text { Aparência de dentes e } \\
\text { Gengiva }\end{array}$} & \multicolumn{2}{|c|}{ Adequada } & \multicolumn{2}{|c|}{ Inadequada } & \multirow[b]{2}{*}{$P$} \\
\hline & & $\mathbf{n}$ & $\%$ & n & $\%$ & \\
\hline \multicolumn{7}{|c|}{$\begin{array}{l}\text { Utilização de serviço } \\
\text { odontológico alguma vez na vida }\end{array}$} \\
\hline & Sim & 226 & 75,1 & 75 & 24,9 & 1,00 \\
\hline & Não & 18 & 75,0 & 6 & 25,0 & \\
\hline \multicolumn{7}{|c|}{ Tempo da última visita ao dentista } \\
\hline & Até 1 ano & 120 & 78,9 & 32 & 21,1 & 0,15 \\
\hline & Mais de 1 ano & 106 & 71,1 & 43 & 28,9 & \\
\hline \multicolumn{7}{|c|}{ Dor de dente } \\
\hline & Não & 71 & 79,8 & 18 & 20,2 & 0,29 \\
\hline & Sim & 173 & 73,3 & 63 & 26,7 & \\
\hline
\end{tabular}




\section{ciência plural}

Não foi observada nenhuma associação estatisticamente significativa entre a variável aparência de dentes e gengiva com as variáveis "utilização de serviço odontológico alguma vez na vida", "tempo da última visita ao dentista" e "dor de dente". No entanto, quando a variável independente é a mastigação, ocorre alteração no comportamento das variáveis conforme observado na Tabela 3.

Tabela 3: Associação entre mastigação e utilização do serviço odontológico, tempo da última visita ao dentista e dor de dente em adolescentes e adultos jovens, Sobral - CE, 2012.

\begin{tabular}{|c|c|c|c|c|c|}
\hline \multirow{2}{*}{$\begin{array}{ll}\text { Variáveis } & \text { Mastigação }\end{array}$} & \multicolumn{2}{|c|}{ Adequada } & \multicolumn{2}{|c|}{ Inadequada } & \multirow{2}{*}{$P$} \\
\hline & $\mathrm{N}$ & $\%$ & $\mathrm{n}$ & $\%$ & \\
\hline \multicolumn{6}{|l|}{$\begin{array}{l}\text { Utilização de serviço odontológico } \\
\text { alguma vez na vida }\end{array}$} \\
\hline Sim & 367 & 91,1 & 36 & 8,9 & 0,72 \\
\hline Não & 28 & 87,5 & 4 & 12,5 & \\
\hline \multicolumn{6}{|l|}{ Tempo da última visita ao dentista } \\
\hline Até 1 ano & 183 & 93,4 & 13 & 6,6 & 0,16 \\
\hline Mais de 1 ano & 184 & 88,9 & 23 & 11,1 & \\
\hline \multicolumn{6}{|l|}{ Dor de dente } \\
\hline Não & 116 & 96,7 & 4 & 3,3 & 0,01 \\
\hline Sim & 279 & 88,6 & 36 & 11,4 & \\
\hline
\end{tabular}

Não foi observada associação estatisticamente significativa entre mastigação e utilização de serviço odontológico alguma vez na vida e tempo da última visita ao dentista. Já a associação entre a mastigação e a dor de dente apresentou-se estatisticamente significativa.

A associação entre a necessidade de tratamento autorreferida e utilização de serviço odontológico alguma vez na vida, tempo da última visita ao dentista e dor de dente se comportou conforme demonstrado na Tabela 4. 


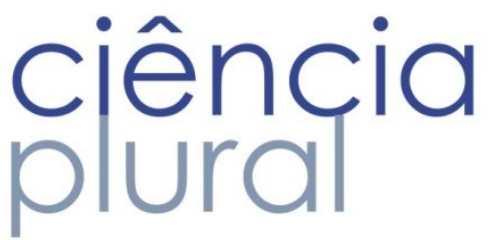

Tabela 4: Associação entre necessidade de tratamento autorreferida e utilização do serviço odontológico, tempo da última visita ao dentista e dor de dente em adolescentes e adultos jovens, Sobral - CE, 2012.

\begin{tabular}{|c|c|c|c|c|c|}
\hline \multirow{2}{*}{$\begin{array}{ll}\text { Variáveis } & \begin{array}{r}\text { Necessidade tratamento } \\
\text { autorreferida }\end{array}\end{array}$} & \multicolumn{2}{|c|}{ Não } & \multicolumn{2}{|c|}{ Sim } & \multirow[b]{2}{*}{ p ajustado } \\
\hline & $\mathrm{n}$ & $\%$ & $\mathrm{n}$ & $\%$ & \\
\hline \multicolumn{6}{|l|}{$\begin{array}{l}\text { Utilização de serviço odontológico } \\
\text { alguma vez na vida }\end{array}$} \\
\hline Sim & 140 & 31,5 & 304 & 68,5 & 0,006 \\
\hline Não & 20 & 55,6 & 16 & 44,4 & \\
\hline \multicolumn{6}{|l|}{ Tempo da última visita ao dentista } \\
\hline Até 1 ano & 65 & 29,0 & 159 & 71,0 & 0,29 \\
\hline Mais de 1 ano & 75 & 34,1 & 145 & 65,9 & \\
\hline \multicolumn{6}{|l|}{ Dor de dente } \\
\hline Não & 58 & 44,6 & 72 & 55,4 & 0,002 \\
\hline Sim & 102 & 29,1 & 248 & 70,9 & \\
\hline
\end{tabular}

Foram observadas associações significativas entre necessidade de tratamento autorreferida e utilização de serviços odontológicos alguma vez na vida e dor de dente.

\section{Discussão}

As condutas e hábitos adotados na vida de uma pessoa são resultados das suas práticas sociais, sabedoria, valores, cultura e conhecimento popular acumulado ${ }^{9}$. Nesse contexto, a percepção em saúde reflete as experiências subjetivas dos indivíduos e retrata 0 nível real de saúde e experiências anteriores com 0 sistema de saúde e serviços específicos recebidos ${ }^{3}$.

Com uma análise dessa concepção, é possivel compreender o referencial dessa população sobre 0 processo saúde-doença. Sendo assim, a autopercepção em saúde incorpora um papel imprescindível para entender a visão do usuário sobre os serviços odontológicos e a sua real demanda.

Não há como versar sobre a autopercepção em saúde bucal sem incluir a representação atribuída à boca humana. Sabe-se que este órgão apresenta funções diversas desde a manducação, a linguagem e 0 erotismo ${ }^{10}$.

Werneck ${ }^{11}$ destacou que no cotidiano do Sistema Único de Saúde (SUS) as práticas concentram-se em torno de uma bucalidade parcial e tímida, não autorreferida e não refletida, havendo, assim, uma negligência do 


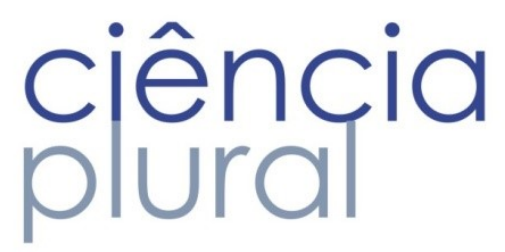

caráter subjetivo no modelo de trabalho e uma ênfase no aspecto clínico. Como consequência, o processo de trabalho em saúde bucal torna-se uma reprodução de modo padronizado ${ }^{11}$, desrespeitando, assim, a bucalidade dos sujeitos envolvidos e as experiências inerentes de cada usuário.

A compreensão dos cirurgiões-dentistas sobre a autopercepção em saúde bucal e bucalidade, nesse contexto, pode ser considerada uma ferramenta importante para a solução desse impasse, visto que a autopercepção em saúde bucal é um importante indicador e mediador entre os sintomas e sinais clínicos de doenças bucais e impactos na qualidade de vida. Além disso, revela informações importantes para 0 planejamento dos serviços odontológicos, pois indica como a rotina e a qualidade de vida da população estão sendo afetadas por essa questão?

Dos indivíduos pesquisados $25,3 \%$ classificaram sua saúde bucal como inadequada. 0 levantamento epidemiológico SB Brasil 2010 obteve um resultado semelhante para a faixa etária de adolescentes, no qual 22,7\% consideraram sua saúde bucal como ruim ou péssima ${ }^{7}$. Esse quadro de predominância da satisfação da condição bucal não condiz com o quadro clínico encontrado na população, visto que apenas 10,4\% estavam livres de cárie. Podendo assim, inferirmos que a concepção subjetiva é menos exigente que o parecer clínico do cirurgião-dentista.

Batista et al. ${ }^{12}$ após realizarem um estudo transversal com 1.824 adolescentes entre 15 a 19 anos no Estado de São Paulo, encontraram que 18,4\% dos participantes consideraram que a aparência dos seus dentes e gengiva afetava seu relacionamento com outras pessoas, destes apenas 9,5\% eram livres de cárie e a média de dentes saudáveis era de 22,2. No presente estudo, embora houvesse um percentual um pouco maior de adolescentes livres de cárie $(10,4), 75,1 \%$ avaliaram que a aparência dos seus dentes e gengiva estava entre boa ou ótima e $48,5 \%$ relataram que o seu relacionamento interpessoal era afetado pela sua saúde bucal, destacando, assim, um maior desconforto com esses aspectos. Esse resultado, pode ser decorrente de um maior discernimento sobre essa relação ou o fato de 42,7\% possuírem alterações oclusais.

Esse quadro de insatisfação se torna mais relevante quando se analisa a associação entre classificação da saúde bucal autorreferida e as outras variáveis relativas à autopercepção em saúde bucal. Os participantes desse estudo que relataram inadequação relativa à aparência de dentes e gengiva, mastigação e necessidade de tratamento tenderam a classificar a sua saúde bucal negativamente.

Em relação à estética dos dentes, em estudo de Gabin et al. ${ }^{13}$ observa-se que 16,2\% dos adolescentes entrevistados responderam que para eles saúde bucal estava relacionada com a aparência dos dentes. 


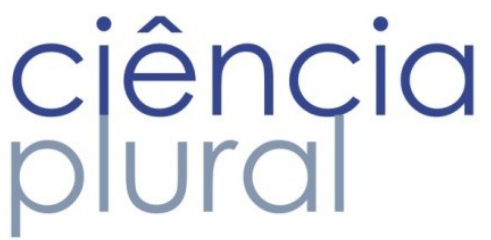

Corroborando com isso, Elias et al. ${ }^{14}$ por meio de uma pesquisa qualitativa com adolescentes de diferentes estratos sociais de Ribeirão Preto constataram que o que mais motivava-os a procurar cuidados de saúde bucal eram fatores associados a estética como aparência pessoal, sexualidade, emprego e da saúde em geral. Esse fato está diretamente ligado com a importância da autoestima e capacidade de se relacionar para essa população. Já em nosso estudo, a aparência de dentes e gengiva não foi evidenciada como motivo determinante para a procura por serviços odontológicos, nem tampouco em relação à dor de dente.

Essa falta de associação entre as variáveis de procura ao atendimento odontológico com a aparência de dentes e gengiva confronta a ideia inicial de que quanto mais insatisfeito com sua aparência bucal, maior a procura por serviços odontológicos a fim de resolver esse problema. Uma explicação plausível para esse fato é a dificuldade de acesso ao serviço de saúde, ou ainda, uma conscientização precária sobre a importância desse cuidado.

Quando analisamos aspectos relativos à mastigação, os participantes que já passaram por algum episódio de dor de dente relataram mais inadequação em relação à mastigação quando comparado aos que não tiveram dor de dente corroborando nossos achados com o estudo de Barbosa et al. ${ }^{15}$. Esse resultado é facilmente explicado pelo aspecto incapacitante da dor de dente.

Identificar a autopercepção sobre saúde é um grande auxílio para compreender o padrão de procura por um serviço de saúde. Para entender o acesso ao serviço é necessário perceber que este apresenta relações com as necessidades, a demanda e a utilização dos serviços. As necessidades em saúde tornam-se demanda que por sua vez, gera a utilização dos serviços, desde que seja possível o acesso ${ }^{16}$.

Seguindo esse contexto, segundo Sheiham e Tsakos ${ }^{17}$ esse conhecimento sobre as necessidades de cuidado à saúde quando bem empregado pode proporcionar influências nas políticas públicas para que estas sejam mais eficientes, acessando o impacto das doenças e necessidades da população local compreendendo as prioridades desses indivíduos.

Pensando nisso, essa pesquisa associou necessidade de tratamento autorreferida com variáveis referentes à utilização de serviço odontológico, tempo da última visita ao dentista e dor de dente. Foi encontrada associação entre necessidade de tratamento autorreferida com a utilização de serviços odontológicos alguma vez na vida e dor de dente. Esse achado referente ao serviço odontológico corrobora com a pesquisa realizada por Machado et al. ${ }^{18} \mathrm{em}$ adultos jovens. 


\section{ciência plural}

No presente estudo, quando se analisa a necessidade de tratamento autorreferida, os participantes que já sentiram dor de dente alguma vez na vida referiam necessitar de tratamento odontológico mais do que os que nunca sentiram dor de dente, o que é condizente com a literatura ${ }^{19}$.

A relação entre a dor de dente e esses dois aspectos é facilmente justificada quando se considera a incapacitação decorrente da odontalgia. $O$ incômodo causado pela dor de dente provoca dificuldades para se alimentar, dormir e realizar atividades cotidianas. Sendo, assim, é compreensível que pacientes que já sentiram esse estímulo doloroso tendam a relatar a necessidade de tratamento ${ }^{20}$.

Nesse contexto, os estudos com os adolescentes tornam-se importantes para compreender melhor as vivências, expectativas e visão do mundo e o modo como buscam ajuda. Esse conhecimento, por sua vez, tem o papel de auxiliar e orientar a criação de medidas mais eficazes e o planejamento de ações educativas e preventivas voltadas para esse grupo ${ }^{21}$.

São necessários estudos futuros que incluam a associação desses aspectos subjetivos com componentes normativos, adquiridos por meio do exame clínico para um melhor entendimento da saúde bucal dessa faixa etária.

\section{Conclusões}

A percepção sobre saúde bucal na faixa etária de adolescentes e adultos jovens em Sobral-CE está relacionada com a estética, função mastigatória e a necessidade de tratamento.

A relação da necessidade de tratamento autorreferida com a utilização de serviços odontológicos e dor de dente, enfatiza o distanciamento dessa população da busca por procedimentos preventivos. Considerando essa perspectiva e a cultura de extração dentária como solução principal para o problema de dor de dente ainda presente em alguns serviços de saúde, seria interessante que as ações educativas promovidas pela equipe de saúde bucal abordassem as várias possibilidades de solução do problema desde a promoção da saúde até a reabilitação oral.

Seria importante, ainda, que o planejamento em saúde bucal fosse orientado não somente por dados normativos, mas que incluísse a percepção da população para que as ações propostas fossem mais coerentes e aceitáveis pelos usuários. Para o avanço dessa proposta seria fundamental repensar o modelo de trabalho vigente, implantando componentes subjetivos nesse processo. 


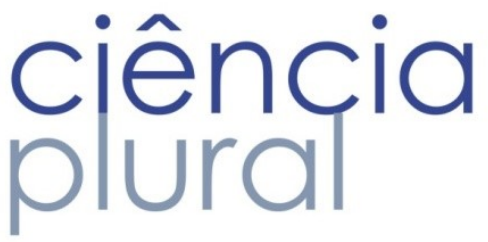

Referências

1. Mendonça HLC, Szwarcwald CL, Damacena, GN. Autoavaliação de saúde bucal: resultados da Pesquisa Mundial de Saúde - atenção básica em quatro municípios do Estado do Rio de Janeiro, Brasil, 2005. Cad. Saúde Pública. 2012; 28(10):1927-1938.

2. Ofstedal MB, Zimmer Z, Cruz G, Chan A, Lin YH. Self-assessed health expectancy among older Asians: a comparison of Sullivan and multistate life table methods. Ann Arbor: University of Michigan, Population Studies Center; 2002.

3. Gift HC, Atchison KA, Drury TF. Perceptions of the natural dentition in the context of multiple variables. J Dent Res 1998; 77(7):1529-1538.

4. Gabardo MCL, Moysés ST, Moysés S. Autopercepção de saúde bucal conforme o Perfil de Impacto da Saúde Bucal (OHIP) e fatores associados: revisão sistemática. Rev Panam Salud Publica 2013; 33(6):439-45.

5. Instituto Brasileiro de Estatística e Geografia (IBGE). Cidades: Sobral, CE. [acesso em 2013 jun 21] Disponível em: http://cod.ibge.gov.br/232RH.

6. WHO - World Health Organization. Oral health surveys: basic methods. 4 ed. Geneva:ORH/EPID, 1997. 7. BRASIL. Ministério da Saúde. Secretaria de Atenção á Saúde. SB Brasil: Projeto Pesquisa Nacional de Saúde Bucal: resultados principais. Brasília: Ministério da Saúde, 2011. [online] [acesso em 2015 nov 25]. Disponível em http://dab.saude.gov.br/CNSB/sbbrasil/arquivos/projeto_sb2010_relatorio_final.pdf

8. BRASIL. Ministério da Saúde. Coordenação Nacional de Saúde Bucal. SB Brasil 2010 - Pesquisa Nacional de Saúde Bucal: Manual da equipe de campo. Brasília, 2009. [online] [acesso em 2015 nov 25]. Disponível em http://dab.saude.gov.br/CNSB/sbbrasil/arquivos/SBBrasil2010_Manual_Equipe_Campo.pdf

9. Oliveira WF, Forte FDS. Construindo o Significado da Saúde Bucal a Partir de Experiência com Mães. Pesq Bras Odontoped Clin Integr 2011; 2(11):183-191.

10. Botazzo C. Cultura, sexualidade, repressão. In: Botazzo C. Diálogos sobre a boca. São Paulo: Hucitec, 2013. p. 90-126.

11. Werneck, MAF. Bucalidade e existência. Ciênc. saúde coletiva 2006; 11(1):28-31.

12. Batista MJ, Rihs LB, Gonçalo CS, Miyauchi kubo FMM, Amaral RC, Sousa MLR. Treatment needs and self-perception of oral health among adolescents. RGO - Rev. Gaúch. Odontol. 2012; 60(3):289-296.

13. Garbin CAS, Garbin AJl, Moimaz SAS, Gonçalves PE. A saúde na percepção do adolescente. Physis 2009; 19(1):227-238.

14. Elias MS, Cano MAT, Mestriner Jr W, Ferriani, MGC. A importância da saúde bucal para adolescentes de diferentes estratos sociais do município de Ribeirão Preto. Rev. Latino-Am. Enfermagem 2001; 9(1):88-95. 


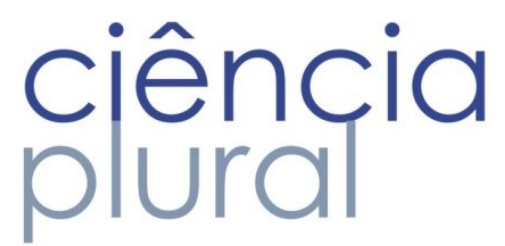

15. Barbosa TB, Junqueira SR, Frias AC, Araujo ME. Interferência da saúde bucal em funções biológicas e sociais segundo a percepção de adolescentes brasileiros. Pesq Bras Odontoped Clin Integr 2013; 13(2):171176.

16. Manhães ALD, Costa AJL. Acesso a e utilização de serviços odontológicos no Estado do Rio de Janeiro, Brasil, em 1998: um estudo exploratório a partir da Pesquisa Nacional por Amostra de Domicílios. Cad. Saúde Pública 2008; 24(1):207-218.

17. Sheiham A, Tsakos G. Oral health needs assessments. In: Pine C, Harris R, editors. Community oral health. Mew Malden:Quintessence Publishing, 2007. p. 59-79.

18. Machado LP, Camargo MBJ, Jeronymo JCM, Bastos GAN. Uso regular de serviços odontológicos entre adultos e idosos em região vulnerável no sul do Brasil. Rev. Saúde Pública 2012; 46(3):526-533.

19. Noro LRA, Roncalli AG, Mendes Júnior FIR, Lima KC, Teixeira AKM. Toothache and social and economic conditions among adolescents in Northeastern Brazil. Ciênc. saúde coletiva 2014; 9(1):105-113.

20. Pau A, Croucher RE, Marcenes W. Demographic and socio-economic correlates of dental pain among adults in the United Kingdom, 1998. Br Dent J 2007; 202(9):E21.

21. Palazzo LS, Bérla JU, Tomasi E. Adolescentes que utilizan servicios de atención primaria? Cómo viven? Por qué buscan ayuda y cómo se expresan? Cad. Saúde Pública 2003; 19(6):1655-1665. 